\title{
SPECTRA AND APPROXIMATIONS OF A CLASS OF SIGN-SYMMETRIC MATRICES
}

\author{
OlgA Y. KuSHEL
}

Abstract. A new class of sign-symmetric matrices is introduced in this paper. Such matrices are called $J$-sign-symmetric. The spectrum of a $J$-sign-symmetric irreducible matrix is studied under the assumption that its second compound matrix is also $J$-sign-symmetric. The conditions for such matrices to have complex eigenvalues on the spectral circle are given. The existence of two positive simple eigenvalues $\lambda_{1}>\lambda_{2}>0$ of a $J$-sign-symmetric irreducible matrix $A$ is proved under some additional conditions. The question when the approximation of a $J$-sign-symmetric matrix with a $J$-sign-symmetric second compound matrix by strictly $J$ sign-symmetric matrices with strictly $J$-sign-symmetric second compound matrices is possible is also answered in this paper.

Mathematics subject classification (2010): Primary 15A48; Secondary 15A18, 15A75.

Keywords and phrases: Totally positive matrices, sign-symmetric matrices, irreducible matrices, compound matrices, exterior powers, Gantmacher-Krein theorem, eigenvalues.

\section{REFERENCES}

[1] F. R. Gantmacher, M. G. Krein, Oscillation Matrices and Kernels and Small Vibrations of Mechanical Systems, AMS Bookstore, 2002.

[2] Tsoy-Wo MA, Classical Analysis on Normed Spaces, World Scientific Publishing, 1995.

[3] I. M. Glazman, Ju. I. Liubich, Finite-Dimensional Linear Analysis: A Systematic Presentation in Problem Form, MIT Press, 1974.

[4] J. L. KelLEy, General Topology, Birkhäuser, 1975.

[5] K. Kuratovski, Topology, I, II, revised 2nd ed., Academic Press, New York, 1966.

[6] F. Gantmacher, The Theory of Matrices, Volume 1, Volume 2, Chelsea, Publ. New York, 1990.

[7] H. Minc, Nonnegative Matrices, John Wiley and Sons, New York, 1988. 